\title{
Quasi-static analysis of spline coupling under load
}

\author{
J.P. De Vaujany ${ }^{a}$, M. Guingand and J. Teixeira Alves \\ Université de Lyon, INSA-Lyon, LaMCoS UMR 5259, 69100 Villeurbanne, France
}

Received 2 December 2015, Accepted 15 March 2016

\begin{abstract}
This paper presents a procedure for analyzing the instantaneous loaded contact of the spline joints. The process permits very fast computation of load sharing, including bending and contact effects. It uses the method of the influence coefficients resolving the equation of compatibility of the displacements. A study of the numerical model allows to show the influence of the deformation of the shafts and of the mounting errors on the contact pressure distribution.
\end{abstract}

Key words: Spline / contact pressure / load sharing

\section{Introduction}

Splines are used in mechanical drive systems to transfer rotatory motion from an input to an output. There are multiple applications for splines in mechanical drive systems which vary from the simple to the complex. In the bibliography, papers present studies with different FEM computations to determine the pressure distribution on the tooth flank $[1,2]$. In order to accelerate the computations, Barrot et al propose an analytical method that is used to investigate the distortions of involute [3,4]. The coefficients of the model have been calculated and benchmarked referring to a FEM model.

Cuffaro et al. [5, 6] studied the pressure distribution on spline coupling teeth that has been investigated by means of analytical approaches and experimental techniques. The experimental pressure distribution has been obtained thanks to a special film, capable of changing color intensity with pressure variation.

In this paper, the proposed method can quickly calculate the contact pressure distribution taking into account the geometry of the shafts and different errors (manufacturing and assembling). A software has been developed to analyse the geometry and the quasi-static behaviour of external and internal splines. To do those analyses, it is necessary to provide the general geometrical informations of the splines and also the conditions of functionality.

One of the objectives was to propose a numerical model to simulate the quasi-static behavior of the spline

\footnotetext{
${ }^{a}$ Corresponding author:

Jean-pierre.devaujany@insa-lyon.fr
}

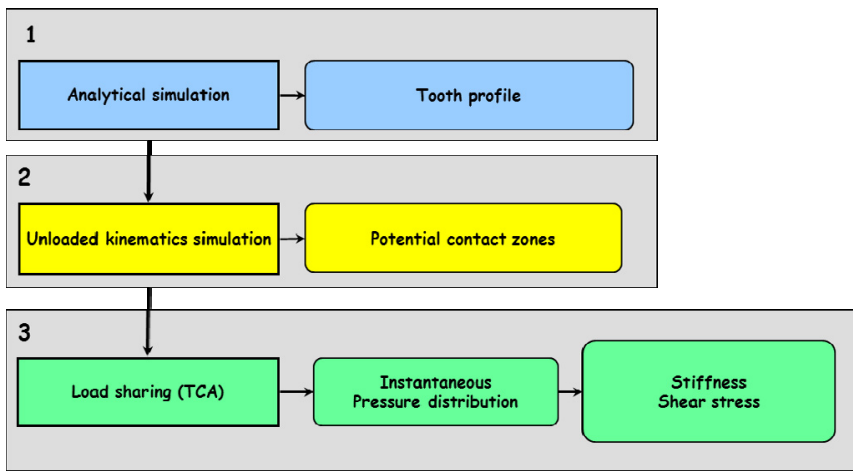

Fig. 1. Analysis procedure.

joints. The objectives of this paper are to present the computational model of a spline and to quantify the influence of the shaft geometry and of the assembly errors on the contact pressure results.

\section{Theoretical analysis}

The procedure for analyzing a spline joint under torque was classically divided into three main steps (Fig. 1). The first one simulates manufacturing to obtain the tooth profiles of the teeth. The second one is an unloaded kinematics simulation to determine the potential contact zones, while the last one is the computation of the load sharing between all the teeth in contact. This stage comprises the computation of instantaneous pressure distribution and meshing stiffness. 


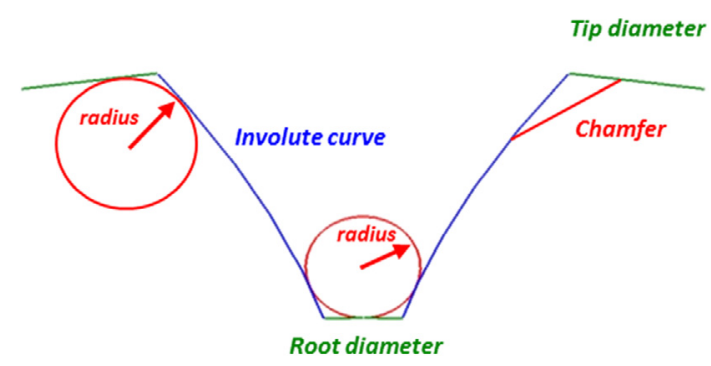

Fig. 2. Spline profile.
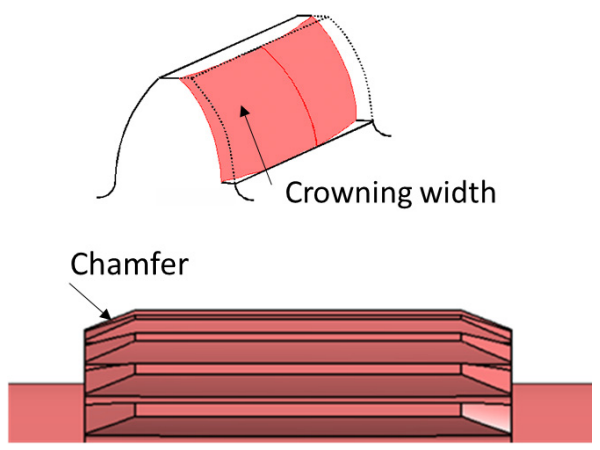

Fig. 3. Crowning width and chamfer.

\subsection{Spline profile and unloaded kinematics simulation}

Respect to the standard ANSI B92.1 - 1996 [7], the flank profile shape is an involute one. Profile modifications can be integrated: chamfer or fillet at the top (Fig. 2) and crown along the tooth width (Fig. 3). The tooth root can be defined by a radius or only by the root diameter. At this step, different manufacturing errors can be integrated on each profile of the teeth (pitch error, helical error and local profile errors on the flank). The same global error on all the teeth or independent errors for each tooth can be imposed.

The 3D geometry is generated automatically in a CAD software CATIA. Shafts (Fig. 4) can be integrated in the CAD part geometry, to take into account their deformation later in the calculation. In the step 2 of the process (Fig. 1), the potential contact points are determined computing the gaps between the flank surfaces of the external and internal splines. It is possible to integrate assembling errors. Figure 4 presents also the direction of the axes of the 4 errors: 2 radial errors along the $X$ and $Y$ axes, one axial error along the $Z$ axis and angular error about the $X$ axis. Then, these potential contact zones are used to calculate the load sharing.

\subsection{Load sharing and pressure distribution}

The last and final step of the process is the loaded TCA (tooth contact analysis) (Fig. 1). The determination of tooth load sharing is above all a multi-contact problem. Potential contact zones are found and the load sharing in these areas must satisfy the displacement compatibility conditions of every point $k[8]$.
On the potential contact area, load sharing is sought to satisfy this equation:

$$
\begin{aligned}
& \left\{\begin{array}{l}
y_{i}^{\text {ang }}=U_{1 i}^{\text {ang }}+U_{2 i}^{\text {ang }}+e i_{i}^{\text {ang }}-\alpha^{\text {ang }}=0 \\
p_{i} \geqslant 0
\end{array}\right. \\
& \text { on the contact area. } \\
& \begin{array}{r}
\begin{array}{l}
y_{i}^{\text {ang }}=U_{1 i}^{\text {ang }}+ \\
p_{i}=0
\end{array} U_{2 i}^{\text {ang }}+e i_{i}^{\text {ang }}-\alpha^{\text {ang }} \geqslant 0 \\
\text { out of the contact area }
\end{array}
\end{aligned}
$$

These two systems of equations can be reduced in just one relation:

$$
y_{i}^{\text {ang }} \cdot p_{i}=0
$$

With :

- $U_{1 i}^{\text {ang }}$ and $U_{2 i}^{\text {ang }}$ are angular displacements of the bodies 1 and 2 at the point $i$,

$-e i_{i}^{\text {ang }}$ is the initial angular gap at the point,

$-\alpha^{\text {ang }}$ is the global displacement of the contact area,

$-p_{i}$ is the pressure at the point $i$.

These parameters, with the final gap $e f_{i}^{\text {ang }}$, are presented in Figure 5. The set of the angular values is defined depending on the rotation axis of the internal splines.

The computation is carried out simultaneously on all the teeth (Fig. 6). In order to calculate the displacements, it is necessary to split the contact zones to obtain local meshing (Fig. 7). These calculations are performed on a local rectangular mesh defined in the tangent plane between the teeth of the internal and external splines.

The pressures calculated have to balance the applied global torque:

$$
\text { Torque }=\sum_{i=1}^{N} R_{i} F_{i}=\sum_{i=1}^{N} R_{i}\left(p_{i} S_{i} r_{i}^{\mathrm{proj}}\right)
$$

with:

$-S_{i}$ is the surface of the rectangle $i$,

$-p_{i}$ is the pressure on the rectangle $i$,

- $R_{i}$ is the radius (distance between the point $i$ and the axis of rotation of the internal splines),

$-r_{i}^{\text {proj }}$ is the ratio to project the load from the reference normal $\overrightarrow{n_{\text {ref }}}$ to lever arm $\overrightarrow{n_{\text {rotat }, i}}$. Then: $r_{i}^{\text {proj }}=\overrightarrow{n_{\text {ref }}}$. $\overrightarrow{n_{\text {rotat }, i}}$.

Furthermore, the elastic properties of the elements permit writing the displacement of point $i$ of an element $k$ such that:

$$
U_{k i}=\sum_{j=1}^{N} C_{i j}^{k} p_{j}
$$

where $C_{i j}^{k}$ are the influence coefficients of element $k$. Considering both elements, we can write $C_{i j}=C_{i j}^{1}+C_{i j}^{2}$, therefore the displacements can be expressed by:

$$
U_{i}=U_{1 i}+U_{2 i}=\sum_{j=1}^{N} C_{i j} p_{j}
$$



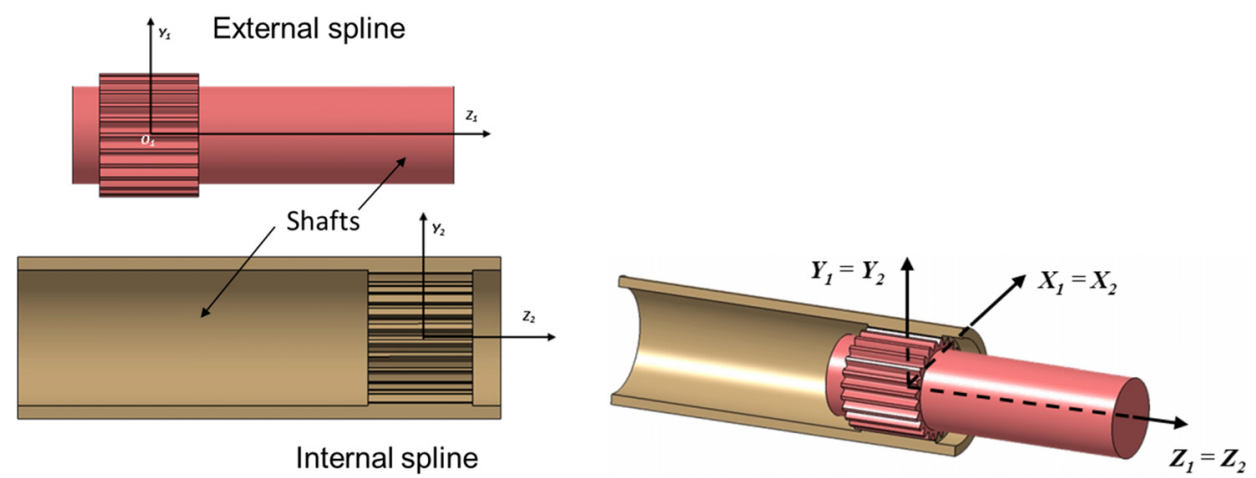

Internal spline

Fig. 4. Spline profile with shafts.

\begin{tabular}{|c|c|c|}
\hline $\begin{array}{l}\text { a) Kinematics contact without } \\
\text { load }\end{array}$ & $\begin{array}{l}\text { b) Displacements and gaps } \\
\text { under load }\end{array}$ & $\begin{array}{l}\text { c) Contact under load after } \\
\text { recalibration }\end{array}$ \\
\hline $\begin{array}{l}\text { Internal } \\
\text { Spline }\end{array}$ & & $\begin{array}{l}\text { Internal } \\
\text { spline }\end{array}$ \\
\hline $\begin{array}{l}\text { External } \\
\text { spline }\end{array}$ & & $\begin{array}{l}\text { External } \\
\text { spline }\end{array}$ \\
\hline
\end{tabular}

Fig. 5. Presentation of the displacements.

If the angular distance between of the two bodies at the point $i$ after loading is noted $y_{i}^{\text {ang }}$ (Fig. 5c), this parameter can be expressed by:

$$
\begin{aligned}
y_{i}^{\text {ang }}= & U_{1 i}^{\text {ang }}+U_{2 i}^{\text {ang }}+e i_{i}^{\text {ang }}-\alpha^{\text {ang }}=\frac{\left(U_{1 i}+U_{2 i}\right) r_{i}^{\text {proj }}}{R_{i}} \\
& +e i_{i}^{\text {ang }}-\alpha^{\text {ang }}=\frac{\sum_{j=1}^{N} C_{i j} p_{j}}{\left(\frac{R_{i}}{r_{i}^{\text {proj }}}\right)}+e i_{i}^{\text {ang }}-\alpha^{\text {ang }}
\end{aligned}
$$

With $e f_{i}^{\text {ang }}=y_{i}^{\text {ang }}+\alpha^{\text {ang }}$ (Fig. 5b), the system is:

$$
\left\{\begin{array}{l}
\sum_{i=1}^{N} R_{i}\left(p_{i} S_{i} r_{i}^{\text {proj }}\right)=\text { Torque } \\
e f_{i}^{\text {ang }}=\frac{\sum_{j=1}^{N} C_{i j} p_{j}}{\left(\frac{R_{i}}{r_{i}^{\text {poj }}}\right)}+e i_{i}^{\text {ang }} \\
p_{i}\left(e f_{i}^{\text {ang }}-\alpha^{\text {ang }}\right)=0
\end{array}\right.
$$

After several manipulations, we finally obtain the equation of compatibility of the displacements:

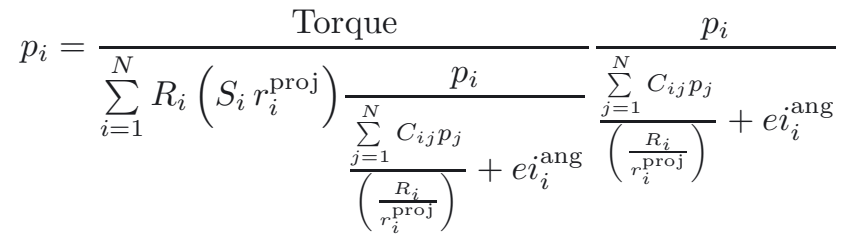

This kind of equations can be solved using an iterative method in order to obtain the value of the pressure at all the points (fixed point iteration method).

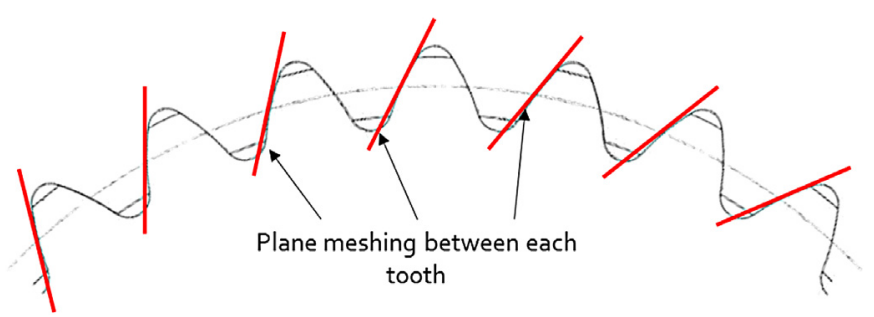

Fig. 6. Meshing between all the teeth.

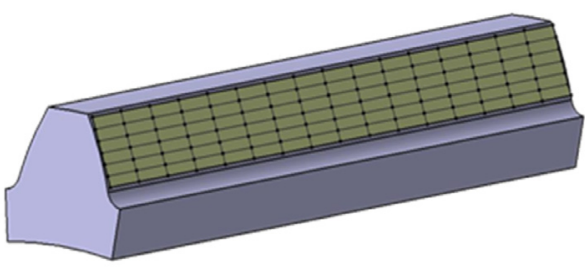

Fig. 7. Local meshing.

The coefficients of influence $C_{i j}$ take into account two kinds of coefficients: the contact coefficients of influence and the bending coefficients of influence. For contact coefficients of influence, since the two solids (internal and external splines) are approximated by two elastic half-spaces, the expression of the displacements is obtained by using the potential functions of Boussinesq and Cerruti [9]. This general expression depends on the combined constants of the two elastic half-spaces, the size of the grid elements of the tangent plane and the coordinates of the grid points. The solution of this integral is known for a rectangular surface, and the displacements at every 


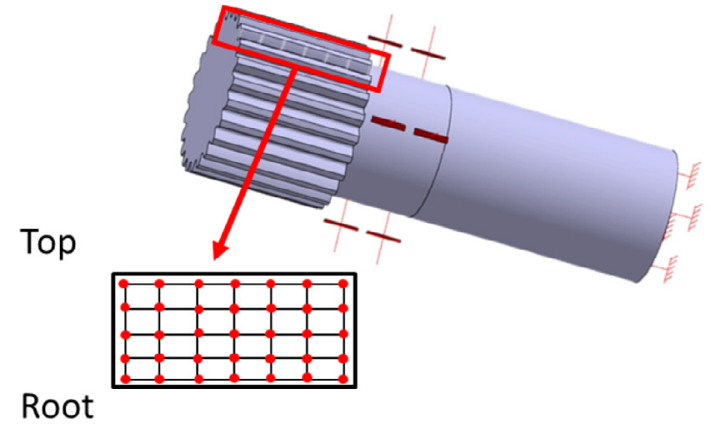

Fig. 8. Conditions for FEM computations.

Table 1. Geometrical characteristics of a spline.

\begin{tabular}{lcc}
\hline & External & Internal \\
\hline Number of teeth & \multicolumn{2}{c}{24} \\
Module $(\mathrm{mm})$ & \multicolumn{2}{c}{1.27} \\
Pressure angle $\left(^{\circ}\right)$ & \multicolumn{2}{c}{30} \\
Face width $(\mathrm{mm})$ & 26 & 26 \\
Profile crown $(\mu \mathrm{m})$ & 20 & \\
Major diameter $(\mathrm{mm})$ & 31.75 & 32.918 \\
Minor diameter $(\mathrm{mm})$ & 27.788 & 29.21 \\
Circular thickness $(\mathrm{mm})$ & 1.995 & 1.995 \\
\hline
\end{tabular}

point $i$ can be computed due to a unit pressure at every point $k$.

The method of defining the bending coefficients of influence is described in several papers $[10,11]$. The different $C_{i j}$ coefficients have been deduced from FEM computations and interpolation functions. These functions were integrated in the calculation process to compute quickly the instantaneous load sharing of the spline. The FEM computations are carried out on nodes equally distributed on the flank of a tooth (Fig. 8). It is possible to integrate the realistic conditions with the geometry of the shaft (length, diameter, variable sections...).

We obtain two types of interpolation functions. It was decided to use $\mathrm{H}$ polynomial functions according to height of the tooth, because the behavior of the tooth along its height is similar to the behavior of a fixed-free beam:

$$
H_{i}(u)=u^{i-1}
$$

Along the tooth width, its behavior is close to the behavior of a free-free beam. That is why we have chosen the resonance functions of such a beam for the $L$ functions:

$L_{j}(v)=\sin \left(\mu_{j} v\right)+\sinh \left(\mu_{j} v\right)-\eta_{j}\left[\cos \left(\mu_{j} v\right)+\cosh \left(\mu_{j} v\right)\right]$

Then, knowing the pressure distribution, the stiffness and the shear stress can also be calculated. The angular stiffness is obtained with the following equation:

$$
K^{\text {ang }}=\frac{\text { Torque }}{\alpha^{\text {ang }}}
$$

The maximum shear stress is a value estimated by the following equation:

$$
\tau_{\max }=0.315 p_{\max }
$$

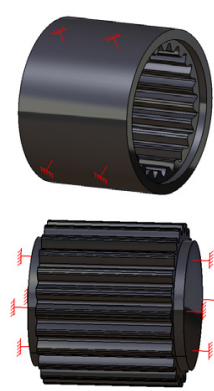

Case 1: without shaft.

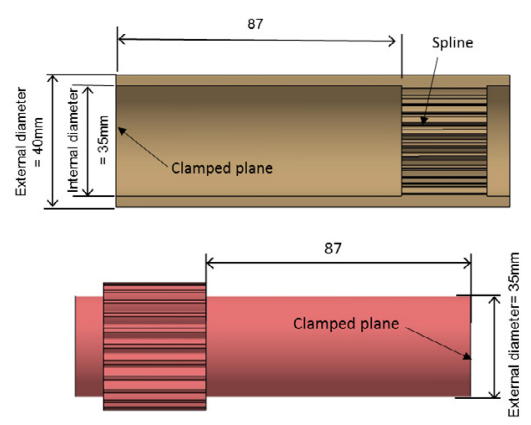

Case 2: with shaft.
Fig. 9. Geometry of shafts and clamping.

The maximum depth is calculated by a chart that gives this value depending on the dimensions of the contact surfaces.

\section{Pressure distribution results}

The general characteristics of the spline used in this work are summarized in Table 1. For the two splines, the tooth root is defined by a fillet radius. The external spline has a fillet at the top tooth $(0.3 \mathrm{~mm})$. The profile crowning is defined on the two sides of the face width. The length and the diameter of the shafts are presented in Figure 9. This figure presents also the clamping conditions at the extremity of the shaft. In the case 1, the two sides of the external splines are clamped, as the external cylinder of the internal spline. In the case 2, only an end of the shaft is clamped. The torque applied is $1500 \mathrm{Nm}$. Young's modulus is $210 \mathrm{GPa}$ and Poisson's ratio is 0.3 for the two splines.

\subsection{Example of results: influence of the geometry of the shaft}

Figure 10 shows the comparison of the pressure distribution on the 24 teeth for the two different cases (Fig. 9). In the case 1 , with a more rigid structure, the pressure distribution is centered on the tooth flanks with a maximum of $798 \mathrm{MPa}$. In the second case, the pressure distribution is delocalized on the side of the face width (946 MPa). The difference between the two maximum pressures is equal to $18 \%$. This difference is explained only by the bending of the shafts.

The load sharing shared on the 24 teeth in contact is similarly the same for the two conditions. Each tooth is loaded with approximately $4735 \mathrm{~N}$.

\subsection{Influence of the mounting errors}

Figure 12 presents the pressure distribution and the load sharing with a radial mounting error along the axis $\mathrm{X}$ (Fig. 11). The error is equal to $0.05 \mathrm{~mm}$. The results 


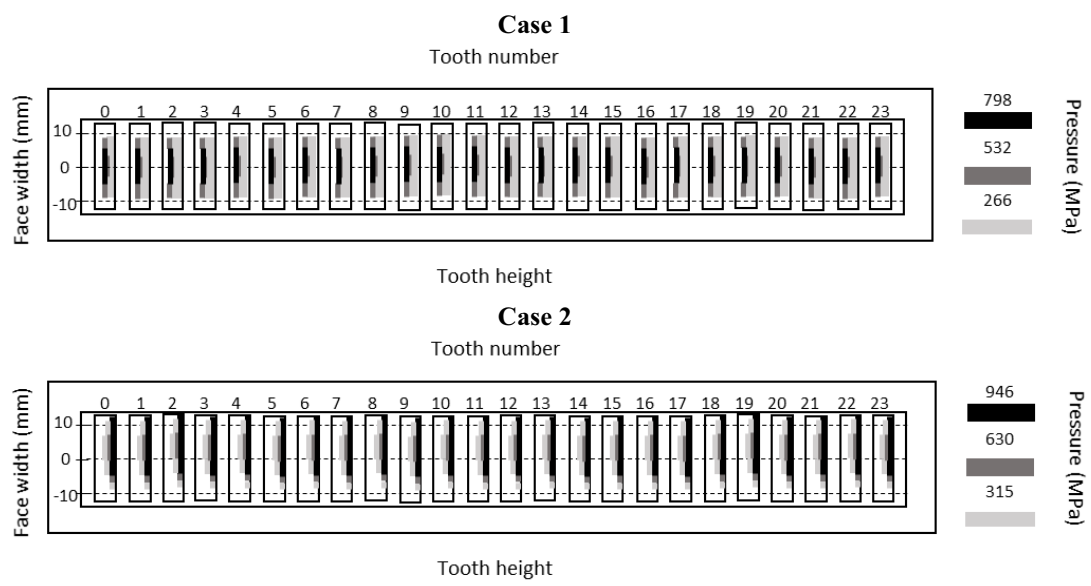

Fig. 10. Pressure distribution for two conditions of shaft geometry.

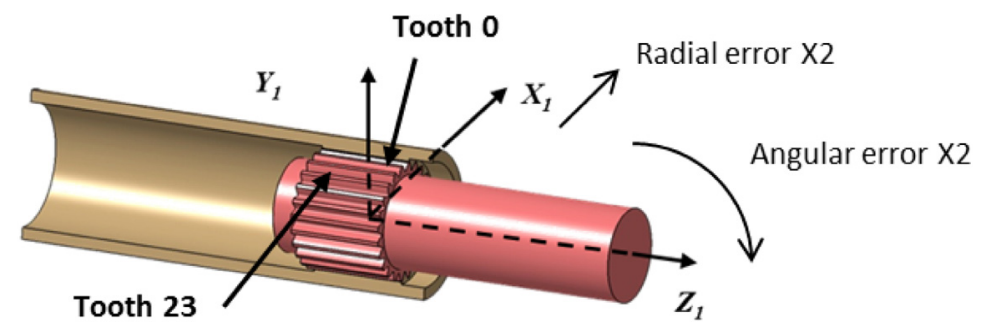

Fig. 11. Tooth angular positions and mounting errors.

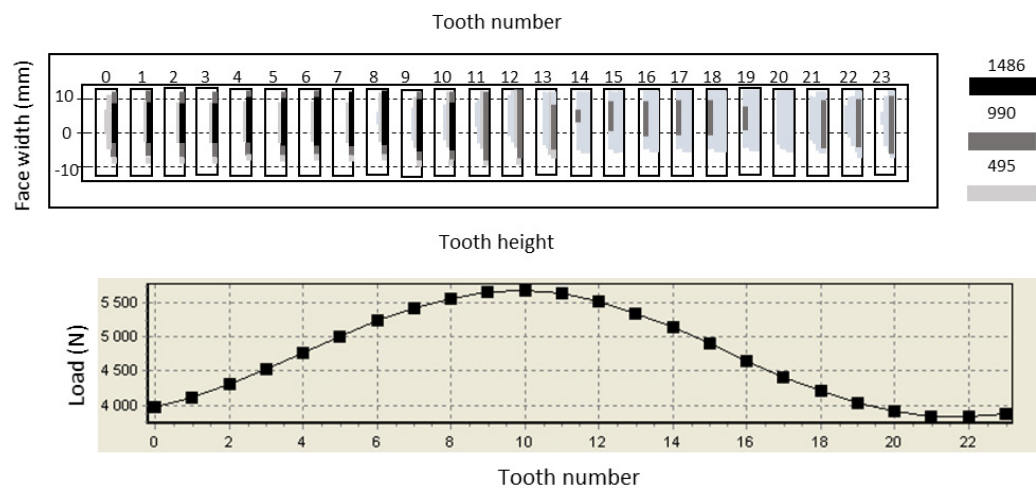

Fig. 12. Pressure distribution and load sharing with $\Delta X=0.05 \mathrm{~mm}$.

are presented only for the most realistic conditions of the case 2 (Fig. 9).

The radial error increases the contact in a zone of the spline (between the teeth 0 and 10). The maximum pressure is $1486 \mathrm{MPa}$. It is a pressure peak on a very small contact surface. In the other part of the spline the pressure are less than $1000 \mathrm{MPa}$, but the contact surfaces are greater than in the other part of the spline. The load sharing shows that the maximum load is $5600 \mathrm{~N}$ on a tooth for a minimum of $4000 \mathrm{~N}$ on another tooth.

Figure 13 presents the pressure distribution and the load sharing with a mounting angular error around the axis X (Fig. 11). The error is equal to 0.1 degree. The results are presented only for the most realistic conditions of the case 2 (Fig. 9). The maximum pressure is
$1061 \mathrm{MPa}$. The load sharing shows that the load varies between $4600 \mathrm{~N}$ and $4900 \mathrm{~N}$.

\section{Conclusion}

In this paper, the pressure distribution along the teeth of spline couplings has been analysed by a numerical model. This one is based on three steps: definition of tooth geometry, kinematics simulation and calculation under load. The latter step is used to calculate the load sharing between the teeth. It takes into account the bending and contact deformations with the method of coefficient of influence. The advantage of this method is its fast computation combined with the accuracy of the results. A 


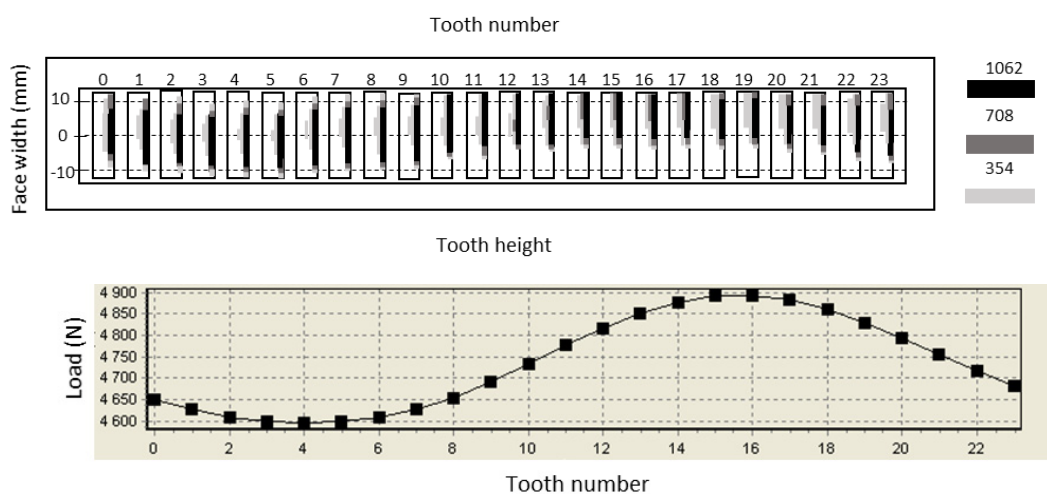

Fig. 13. Pressure distribution and load sharing with $\delta X=0.1$ degree.

study has been presented to show the influence of the geometry of the shafts on the results. The deformation of the shafts and the mounting errors modify the contact pressure distribution. The model allows also to take into account different tooth flank errors.

Acknowledgements. This research work was carried out in the framework with the financial support of Hispano-Suiza, Safran.

\section{References}

[1] J. Hong, D. Talbot, A. Kahraman, Semi-analytical load distribution model for side-fit involute splines, Mech. Mach. Theory 76 (2014) 39-55

[2] J. Hong, D. Talbot, A. Kahraman, Load distribution analysis of clearance-fit spline joints using finite elements, Mech. Mach. Theory 74 (2014) 42-57

[3] A. Barrot, M. Paredes, M. Sartor. Determining both radial pressure distribution and torsional stiffness of involute spline couplings, Proc. IMechE Part C: J. Mech. Eng. Sci. 220 (2006) 1727-1738
[4] A. Barrot, M. Paredes, M. Sartor, Extended equations of load distribution in the axial direction in a spline coupling, Eng. Failure Analysis 16 (2009) 200-211

[5] V. Cuffaro, F. Cura, A. Mura, Test Rig for Spline Couplings Working in Misaligned Conditions, J. Tribol. 136 (2013) 011104-011104-7

[6] V. Cuffaro, F. Cura, A. Mura. Analysis of the pressure distribution in spline couplings. Proceedings of the Institution of Mechanical Engineers, Part C: J. Mech. Eng. Sci. 226 (2012) 2852-2859

[7] Involute Splines Inspection, Standard ANSI B92.1-1996

[8] M. Guingand, J.P. de Vaujany, C.Y. Jacquin, Quasi-static analysis of a face gear under torque, Comput. Methods Appl. Mech. Eng. 194 (2005) 4301-4318

[9] J. Boussinesq, Application des Potentiels à L'étude de L'équilibre et du Mouvement des Solides Élastiques, Albert Blanchard, Paris, 1959, 564 p.

[10] M. Guingand, J.P. De Vaujany, Y. Icard, Fast threedimensional quasi-static analysis of helical gears using the finite prism method, Trans. ASME, J. Mech. Design 126 (2004) 1082-1088

[11] J. Teixeira Alves, M. Guingand, J.P. de Vaujany, Set of functions for the calculation of bending displacements for spiral bevel gear teeth, Mech. Mach. Theory, 2009 